\title{
Some of the Uses of Ultra-Violet Rays in Dermatology
}

\author{
By R. M. B. MacKENNA, M.A., M.D., M.B., B.Ch., F.R.C.P. \\ Physician in Charge, Dermatological Dept., St. Bartholomew's Hospital, London \\ and T. WAREHAM, M.C.S.P. \\ Superintendent of the Physiotherapy Dept., St. Bartholomew's Hospital, London. \\ (With acknowledgement to The British Journal Physical Medicine).
}

\begin{abstract}
TN THIS paper we propose discussing the I.treatment of four skin diseases (psoriasis, cutaneous tuberculosis, acne vulgaris and alopecia areata) with ultra-violet rays, these being the four principal maladies within our purview for which this type of treatment is of benefit.
\end{abstract}

\section{Psoriasis}

There is little doubt that the average psoriatic skin requires a relatively large amount of sun, or ultra-violet ray, for its full health, and many persons prone to this malady keep this disease at bay, if not completely in abeyance, by careful and regular irradiation at home. If the individuals use common sense there seems to be little danger in this custom. The danger of provoking cutaneous precancerosis and epitheliomas is greater in theory than in practice.

When the malady has developed, the Goeckerman Regime, or a modification of this, is by far the most satisfactory method of treatment. Admittedly the Regime is an in-patient one, but can be modified for out-patient treatment, and also for use in the home.

The modification we use for in-patients is as follows:-

White's Tar Paste (National Formulary, 1952), adjusted to contain a variable amount of coal tar in accordance with the type of individual conconcerned, is employed (briefly, other things being equal, the more brunette the patient, the stronger the concentration of tar which can be employed), 4 per cent. is a satisfactory concentration for most skins. The paste is well rubbed into the lesions by hand. Next morning the patient has a tar bath made by adding 2-4 ozs. of liquor picis carbonis or Liquor Carbonis Detergens to 25 gallons of hot water. When in the bath, loose scales are gently removed. Then liquid paraffin is applied to all the areas which are to be exposed to ultra-violet rays. This application of liquid paraffin is an important part of the treatment. Any paste remaining on the skin should be removed with it; the paraffin keeps the horny layer of the epidermis supple, and contrary to the general opinion, unlike vegetable oils, is a highly efficient transmitter of ultra-violet rays.

The details of ultra-violet irradiation are as follows. The day before starting treatment, a test dose is done, so that an exact estimate is made with each patient of the dose required to produce a first degree erythema (E.1.), that is a dose which produces a mild erythema that quickly fades and is not followed by any visible desquamation.

When receiving treatment the patient is completely stripped. The Hanovia "Alpine Sun" Lamp is used at a distance of 18 inches.

The first day, the anterior and posterior aspects of the trunk and legs are irradiated in four areas; the lamp is centred at the xiphoid when treating the trunk, and at the knee when treating the legs. The legs should $\mathrm{n} t$ be covered when irradiating the trunk, or vice versa. The second day, the sides of the trunk and legs are treated, again in four areas; the third day the same areas as on the first day, and so on.

The progression of the dose is most important; a mild E.1. is kept up by increasing the time by 20 per cent. of the previous dose. Stronger doses cause peeling which delays further treatment. Sub-erythema doses have not been found to be nearly as beneficial. Before starting treatment at each attendance, the physiotherapist finds out what reaction was obtained with the previous dose; she enters this in the records and then estimates the next dose in the light of this knowledge. It is obvious that meticulous records must be kept by the physiotherapist.

After each irradiation the tar paste is applied to all the lesions and the patient returns to bed. Every morning the routine of tar bath, liquid paraffin application, ultra-violet irradiation and application of tar paste, is repeated. Autohaemotherapy using 5-10 millilitres of blood is also given once every five days, and some patients benefit if they receive small doses of a sedative.

By the proper use of this method an extensive eruption can be removed in an average period of about 17 days; by this time there is usually some pigmentation of the skin with areas of leucoderma to mark the sites where the lesions have been present.

Note that this regime is dangerous for those having latent or active tuberculosis, because this infection may be accentuated by the irradiation. Therefore full investigation must be made in each case before the regime is commenced.

The principle of tar treatment lies in the fact that tar is a photo-sensitizing agent, and that the beneficial effect of the rays is considerably enhanced by the tar paste and the tar baths; possibly there is more from the biochemical standpoint which can be added, but in the present state of our knowledge it is inadvisable to make further comment. 
It will be obvious to the reader that the treatment can be carried out at home if the patient does not object to the staining of his sheets by the tar paste.

It may be modified for out-patient treatment, but the time required to achieve removal of the lesions is usually longer.

A similar procedure is adopted in out-patient treatment. At each attendance the patient has a tar bath, is then rubbed all over with the liquid paraffin and then is irradiated over four areas. As the irradiation time increases, the total treatment becomes too long, so, in the latter stages only, two areas are treated at each attendance, an accurate E.1. still being obtained by progressing as before. A course may last 6-10 eweks, then a rest is given followed by a further course if necessary. With some patients, a stubborn patch may Iremain when the rest of the skin has cleared; this may be given local ultra-violet ray treatment with considerably stronger doses.

It is interesting that severe concomitant psoriasis of the scalp clears more quickly if treated by giving strong high-frequency sparks to the whole scalp for twenty minutes at each attendance.

\section{Cutaneous tuberculosis}

The treatment of lupus vulgaris and other forms of cutaneous tuberculosis by ultra-violet ray has been so much discussed since the days of Finsen, that we will not make more than a few comments about this matter. Perhaps because of improvement in purity of milk supplies as well as because of better general standards of hygiene and nutrition, our reticence is somewhat stimulated by the fact that this group of diseases, once all too common in this country, is now rare. Further, there is some tendency for the treatment of lupus by ultraviolet ray to be concentrated in a few centres where the Finsen-Lomholt lamp is available; the proper use of this apparatus can be learned only at such a centre, and technical descriptions are available in several standard books. Therefore we will confine our comments to the use of the water-cooled Kromayer lamp, but before doing this it is perhaps worth noting that the most modern treatments of cutaneous tuberculosis (for example calciferol, streptomycin with para-aminosalicylic acid, and derivatives of iso-nicotinic acid), often, particularly with the first named, succeed in clearing wide areas of the infection but leave islets of lupus tissue among the scar tissue. These may be dealt with by chemical cauterization, but in selected cases the Kromayer lamp may be used for this purpose.

If, in the treatment of the infection, it is decided that the Kromayer lamp shall be used the technique we prefer is as follows. Very strong blister doses are given with contact and firm pressure. About five times the minimum dose that would be required to produce a blister is given. Each active area and a margin beyond is treated in rotation and, when all peeling has ceased, a further blister dose is given and repeated to each area four times. A general carbon-arc treatment may also be given at the same time.

If reliance is placed on ultra-violet ray treatment alone, patience and perseverance are required on the part of the patient as treatment may extend over many months.

\section{Acne vulgaris}

Despite the conflicting theories concerning the aetiology of acne vulgaris, it has formerly been recognized that heliotherapy (particularly when associated with sea-bathing) is often reasonably successful in curing or causing amelioration in acne vulgaris in otherwise healthy subjects. Ultraviolet rays derived from a mercury vapour lamp are often more successful for local treatment but therapy with the carbon-arc lamp with its wider spectrum is frequently more beneficial for general treatment.

Probably in the early, comedo, stage, routine dermatological treatment is all that is required; it is in the pustular stage that the rays are most beneficial when properly employed.

It may be helpful to note that mild dermatological treatment of the skin need not be discontinued during the period that ultra-violet ray is given, provided the following two items are remembered:-

(1) The skin must be carefully cleansed of medicament before treatment is employed.

(2) The dermatological treatment must not consist of strongly exfoliative applications, for if these are used the skin will become sore and ultraviolet ray treatment will have to be delayed or modified. Calamine lotion with 2 per cent. precipitated sulphur or a weak sulphur cream are the two remedies which we have found to be of most benefit in these circumstances. The physiotherapeutic technique is, briefly, as follows. Before starting treatment, a test dose is done on a similar area of skin elsewhere on the body. A mild peeling dose is given to these patients (E.2.) and it is important that further irradiation is not given to any area until all peeling has stopped. Therefore each area can only be irradiated about once in each time by increasing the time by about 40 per cent. At the intervening attendances general carbon-arc treatment may be given to other parts of the body using suberythema doses. Some cases benefit by local shortwave diathermy to the peeling areas to increase the local circulation. The technique of irradiation is specialized, as a good cosmetic effect is of first importance. Harsh lines of demarcation from protecting towels must, as far as possible, be avoided.

For example, when treating the face, the skin is well washed with soap and water and any large 
blackheads gently removed. The eyelids are coated with paraffin on to which is placed a wisp of cotton wool; the tips of the ears are lightly smeared with grease and the tip of the nose is dusted with powder (otherwise it tends to receive more rays than the surrounding skin). If the neck is clear of spots, a towel may be wrapped round it and the rest of the body covered. The face is then irradiated without other protection from three aspects; one parallel with the forehead, and two parallel with the zygomatic arches the lamp being centred on a level with the nose.

Similarly, when the back and shoulders are to be treated, only the head and the lower limit of irradiation are covered. The patient sits on a stool and is irradiated in four aspects; two with the lamp parallel with the scapulae at the level of the inferior angles, and two with the lamp above, parallel with the oblique upper fibres of trapezius; usually, the first two areas are treated at one attendance and the second two at the next. This method obviates any unsightly lines where the reaction ceases suddenly at a towel protection line.

\section{Alopecia}

Here we are concerned only with alopecia areata. The cause of this type of baldness is unknown, and the action of ultra-violet rays and the other measures discussed here appears to be solely to stimulate the metabolic activities of the scalp.

We have found that, for many cases, a combination of ultra-violet rays and high frequency sparks is beneficial (though critics would perhaps accuse us of falling into the logical fallacy of arguing post hoc ergo propter hoc).

The technique used is as follows. The skin is first cleansed of grease with methylated ether and then fairly strong peeling doses are given to each patch. As before, further irradiation cannot be given until all peeling has stopped. The patient must continue to attend and is treated by strong high-frequency sparks for twenty minutes at each attendance until the patch is ready for further irradiation.

When the course is complete the ultra-violet ray is stopped but the high-frequency is continued twice a week until, after a suitable interval, another course of ultra-violet ray is given.

It is our custom to allow these patients to continue on treatment for many months, as often three or four courses are necessary. As the patients administer the high-frequency to themselves (under supervision) they take up very little of the physiotherapists' time.

\section{Envoy}

Ultra-violet rays may, in our opinion, be successfully used in four cutaneous maladies, as described above. Because the rays stimulate the metabolic activities of the skin, it may be presumed that they will also increase the powers of self-disinfection of the skin. Cases of persistent furunculosis (not associated with systemic disease) may be successfully treated, particularly with carbon-arc therapy; but we would make no further claims than those in a general outline of the usefulness of ultra-violet ray in dermatology although others, doubtless, would add certain other maladies (for example indolent cutaneous ulceration, indolent wounds and pressure sores) to our brief list.

\section{GENERAL}

We welcome the following new members to the Society: Mrs. Elliott and Miss L. Bradley, both of Port Elizabeth.

Miss Muriel Miller, Miss Mary Gammon, Miss Riva Cohen and Miss Bobrow, all of Johannesburg.

The final year physiotherapy students at Witwatersrand University held their annual dance at the Langham Hotel on September 1st, it was a most enjoyable evening.

Miss A. M. Smith, the part-time paid Secretary employed ty the Society has found it necessary to resign her position. This will be a great loss to the Society, and may we thank her for all that she has done for us in the past year.

Telegrams of good wishes were sent to the World Confederation of Physical Therapy. on the occasion of the First International Congress.

Miss Marjorie Humphrey is acting as Senior Physiotherapist at the Johannesburg General Hospital in the absence of Miss Lois Dyer.

\section{PERSONAL}

Miss Hazel Parframar, M.C.S.P., was married on July 25th, to Mr. Geoffrey Barnes.

Miss Mary Nichol, B.Sc.(Rand.), was married to Mr. Evan Williams in East London on July 13th.

Miss Betty Andrews, B.Sc.(Rand.), was married to Dr. Derrick Dell in Johannesburg, on September 2nd.

May we wish them all every happiness for the future.

Miss Lola Todes has announced her engagement to Mr. Cecil Bersor of Cape Town.

Miss Muriel Miller has also announced her engagement to Mr. Joe Goodman.

\section{EASTER STAMP CAMPAIGN, 1953.}

The efforts of all those who helped with the Easter Stamp Campaign were greatly appreciated by the South African Care of Cripples Association.

Stamps sold by individual Physiotherapists amounted

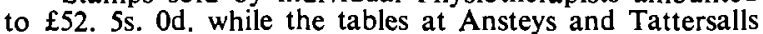
sold $£ 99.12 \mathrm{~s}$. $6 \mathrm{~d}$. and $£ 24$. 3s. Sd. respectively.

These are ever greater amounts than last year.

Thank you all for your help. 